Article

\title{
Spin State Control of the Perovskite Rh/Co Oxides
}

Ichiro Terasaki $^{1, \star}$, Soichiro Shibasaki ${ }^{1}$, Shin Yoshida ${ }^{1}$ and Wataru Kobayashi ${ }^{2}$

${ }^{1}$ Department of Applied Physics, Waseda University, Tokyo 169-8555, Japan

${ }^{2}$ Waseda Institute for Advanced Study, Waseda University, Tokyo 169-8050, Japan

* Author to whom correspondence should be addressed; E-Mail: terra@ waseda.jp;

Tel./Fax.: +81-3-5286-3854.

Received: 30 December 2009; in revised form: 23 January 2010 / Accepted: 26 January 2010 /

Published: 27 January 2010

\begin{abstract}
We show why and how the spin state of transition-metal ions affects the thermoelectric properties of transition-metal oxides by investigating two perovskite-related oxides. In the A-site ordered cobalt oxide $\mathrm{Sr}_{3} \mathrm{YCo}_{4} \mathrm{O}_{10.5}$, partial substitution of $\mathrm{Ca}$ for $\mathrm{Sr}$ acts as chemical pressure, which compresses the unit cell volume to drive the spin state crossover, and concomitantly changes the magnetization and thermopower. In the perovskite rhodium oxide $\mathrm{LaRhO}_{3}$, partial substitution of $\mathrm{Sr}$ for La acts as hole-doping, and the resistivity and thermopower decrease systematically with the $\mathrm{Sr}$ concentration. The thermopower remains large values at high temperatures $(>150 \mu \mathrm{V} / \mathrm{K}$ at $800 \mathrm{~K})$, which makes a remarkable contrast to $\mathrm{La}_{1-x} \mathrm{Sr}_{x} \mathrm{CoO}_{3}$. We associate this with the stability of the low spin state of the $\mathrm{Rh}^{3+}$ ions.
\end{abstract}

Keywords: thermopower; spin state; oxide thermoelectrics; Heikes formula

\section{Introduction}

The spin state is one of the most fundamental concepts in transition-metal compounds/complexes [1]. The Coulomb repulsion from the neighboring oxygen anions changes the $d$ energy levels in transition-metal oxides. In a transition-metal ion surrounded with octahedrally-coordinated oxygen anions, the five-fold degenerate $d$ orbitals in vacuum are split into the triply degenerate $t_{2 g}$ orbitals 
and the doubly degenerate $e_{g}$ orbitals, and the energy gap between the $t_{2 g}$ and $e_{g}$ levels called "ligand field splitting" competes with the Hund coupling. When the ligand field splitting is larger, the $d$ electrons first occupy the $t_{2 g}$ states to minimize the total spin number. On the other hand, when the Hund coupling is strong, the total spin number is maximized. The former state is called "low spin state", and the latter "high spin state". In general, the high spin state is stable at high temperature, because its spin entropy is larger than the entropy of the low spin state.

When the energies of the two spin states are close, various external perturbations such as temperature, pressure and magnetic field can induce the spin state transition/crossover [2]. While the spin state crossover is often observed in transition-metal organic complexes, it is rarely observed in the transition-metal oxides except for cobalt oxides in which the low and high spin states of the $\mathrm{Co}^{3+}$ ion are almost degenerate [3]. $R \mathrm{CoO}_{3}(R$; rare-earth) is a prime example in which the magnetization changes dramatically with temperature and physical/chemical pressure [4,5]. A more complicated issue is the possible existence of the intermediate spin state [6], which is still controversial [7-10].

Since the discovery of the good thermoelectric properties in the layered cobalt oxide $\mathrm{Na}_{x} \mathrm{CoO}_{2}$ [11], oxide thermoelectric materials have been extensively investigated [12]. Unlike the state-of-the-art thermoelectric materials, the carriers in the cobalt oxide feel the spin and orbital degrees of freedom that can contribute to the thermopower [13], as was first proposed by Koshibae et al. [14]. In this article we report why and how the spin states are related to the thermopower of the perovskite-related oxides by studying two prototypical examples, $\mathrm{Sr}_{3} \mathrm{YCo}_{4} \mathrm{O}_{10.5}$ and $\mathrm{LaRhO}_{3}$.

\section{Results and Discussion}

\subsection{Thermopower in correlated systems}

First, we briefly review the physical meaning of the thermopower. According to the Boltzmann equation, the electrical current density $\vec{j}$ and the thermal current density $\vec{q}$ are expressed by the linear combination of the electric field $-\vec{\nabla} V$ and the temperature gradient $-\vec{\nabla} T$ as

$$
\begin{aligned}
& \vec{j}=\sigma(-\vec{\nabla} V)+\sigma S(-\vec{\nabla} T) \\
& \vec{q}=\sigma S T(-\vec{\nabla} V)+\kappa^{\prime}(-\vec{\nabla} T)
\end{aligned}
$$

where $\sigma$ is the conductivity, $S$ is the thermopower, and $\kappa^{\prime}$ is the thermal conductivity for $-\vec{\nabla} E=0$ [15]. In the absence of temperature gradient $-\vec{\nabla} T=0$, we get

$$
\frac{\vec{q}}{T}=S \vec{j}
$$

Considering that the left-hand side of this equation is the entropy current density, one can identify the thermopower $S$ to the entropy per charge when the scattering times involved in $\vec{j}$ and $\vec{q}$ are the same. In this context, the thermopower is a good measure of entropy of carriers, and thus it can detect the entropy due to various degrees of freedom coupled with the carriers.

Koshibae et al. [14] extended the Heikes formula [16] in order to include the spin and orbital degrees of freedom. According to this, the thermopower of the transition metal oxides in the high temperature limit is given by

$$
S=\frac{k_{B}}{e} \log \frac{g_{A}}{g_{B}} \frac{x}{1-x}
$$


where $g_{A}$ and $g_{B}$ are the degeneracies of the $A$ and $B$ ions respectively, and $x$ is the content of the $A$ ions. In the layered cobalt oxide $\mathrm{Na}_{x} \mathrm{CoO}_{2}$, the cobalt ions exist as a mixture of $\mathrm{Co}^{3+}$ and $\mathrm{Co}^{4+}$. The magnetic measurement has revealed that they are in the low spin states at $300 \mathrm{~K}$ [17]. As schematically drawn in Figure 1, the six electrons in the low-spin $\mathrm{Co}^{3+}$ ion fully occupy the $t_{2 g}$ levels, so that it has no other degenerate state. In contrast, in the low-spin $\mathrm{Co}^{4+}$ ion, one electron is removed out of the six electrons, and thus six states are degenerate. Substituting $g_{A}=6$ and $g_{B}=1$ in Equation (4), we evaluate the thermopower to be $k_{B} \log 6 / e=150 \mu \mathrm{V} / \mathrm{K}$. This value is close to the thermopower of $\mathrm{Na}_{x} \mathrm{CoO}_{2}$ at $1000 \mathrm{~K}$ [18]. This is reasonable, because the Heikes formula is an asymptotic expression of the thermopower in the high temperature limit. Here we ignore the $x$ dependent term, because $x$ is close to 0.5. This large entropy is evidenced by the specific heat measurement [19], and the thermodynamic properties of $\mathrm{Na}_{x} \mathrm{CoO}_{2}$ is compared with those of heavy fermion intermetallics [13]. We should further note that Equation (4) explains why all of the related layered cobalt oxides show large thermopower [20-23].

Figure 1. Schematic drawing for the explanation of conduction mechanism of the cobalt oxide proposed by Koshibae et al. [14].

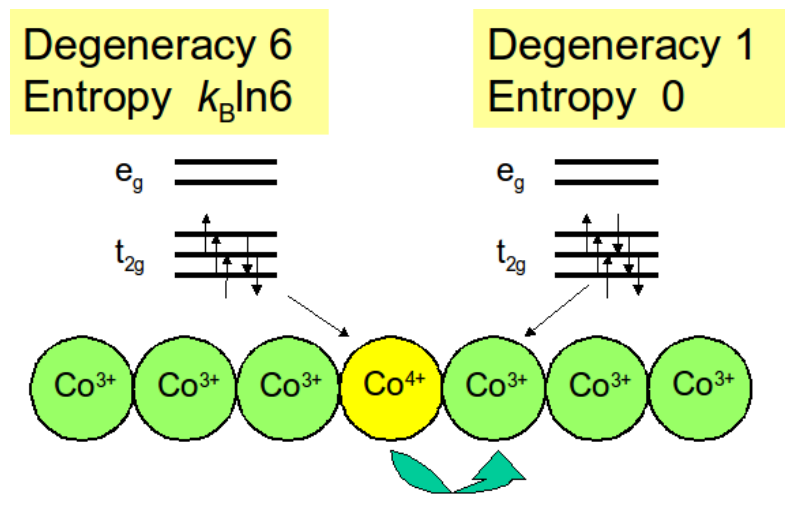

This vividly exemplifies the importance of the spin and orbital entropy stored in the transition-metal

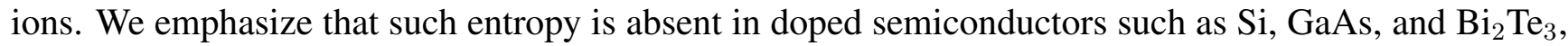
which offers a unique design of thermoelectric materials using the transition metal oxides. Previously Kobayashi et al. [24] showed by controlling the entropy current that the thermopower can be negative for hole-doped semiconducting manganese oxides $\mathrm{CaMn}_{3-x} \mathrm{Cu}_{x} \mathrm{Mn}_{4} \mathrm{O}_{12}$.

\subsection{The A-site ordered perovskite cobalt oxide}

Recently, Kobayashi et al. [25] found that $\mathrm{Sr}_{1-x} \mathrm{Y}_{x} \mathrm{CoO}_{3-y}$ shows a ferromagnetic transition below $340 \mathrm{~K}$ for polycrystalline samples in a limited range of the $\mathrm{Y}$ content from $x=0.20$ to 0.25 . They found that the ferromagnetism is closely related with the ordering of the A-site cations approximately in a ratio of $\mathrm{Sr}: \mathrm{Y}=3: 1$ [26-28]. To emphasize this ordering, we will denote this material $\mathrm{Sr}_{3} \mathrm{YCo}_{4} \mathrm{O}_{10.5}(\mathrm{SYCO})$ in this article. Kobayashi et al. [29] further found various similarities to $\mathrm{LaCoO}_{3}$ in the high-temperature transport above $T_{c}$. A significant difference is that the $\mathrm{CoO}_{6}$ volume is larger in $\mathrm{SYCO}$ than in $\mathrm{LaCoO}_{3}$, and accordingly the high spin state is stable down to low temperatures. This volume is indeed critical, and the magnetism of SYCO is susceptible against chemical and physical pressure [30]. The magnetization 
decreases below $190 \mathrm{~K}$ for some samples, suggesting that a part of the $\mathrm{Co}^{3+}$ ions go to the low spin state. Kimura et al. [31] discovered a metamagnetic transition near $40 \mathrm{~T}$ in such samples, and ascribed this to the spin-state crossover induced by an external magnetic field.

As shown in Figure 2, this particular oxide basically crystallizes in a brownmillerite-like structure, where the octahedral $\mathrm{CoO}_{6}$ layer and the tetrahedral/pyramidal $\mathrm{CoO}_{4.25}$ layer are alternately stacked with insertion of the ordered $\mathrm{Sr}_{0.75} \mathrm{Y}_{0.25} \mathrm{O}$ layer [26,28]. Very recently Sheptyakov et al. [32] have shown that the $\mathrm{Co}$ ions in the $\mathrm{CoO}_{6}$ layer occupy the intermediate spin state, and those in the $\mathrm{CoO}_{4.25}$ layer do the high spin state. This is, however, an oversimplified picture; Ishiwata et al. [33] analyzed the crystal structure of the related oxide $\mathrm{Sr}_{3.1} \mathrm{Er}_{0.9} \mathrm{Co}_{4} \mathrm{O}_{10.5}$ by means of the Rietveld refinement of $\mathrm{X}$-ray diffraction patterns, and found that this oxide exhibits a much more complicated large unit cell with various inequivalent cobalt sites. The same super-structure was observed through the electron microscope by James et al. [34].

Figure 2. Crystal structure of $\mathrm{Sr}_{3} \mathrm{YCo}_{4} \mathrm{O}_{10.5}$. O* represents the oxygen site with $25 \%$ occupancy. The octahedra and tetrahedra correspond to oxygen networks. The structure is brownmillerite-like, and the octahedra and tetrahedra are alternately stacked along the $c$ axis. $\mathrm{Sr}$ and $\mathrm{Y}$ are ordered along the $a b$ plane, and are stacked along the $c$ axis with a periodicity like -Sr-Y-Y-Sr-.

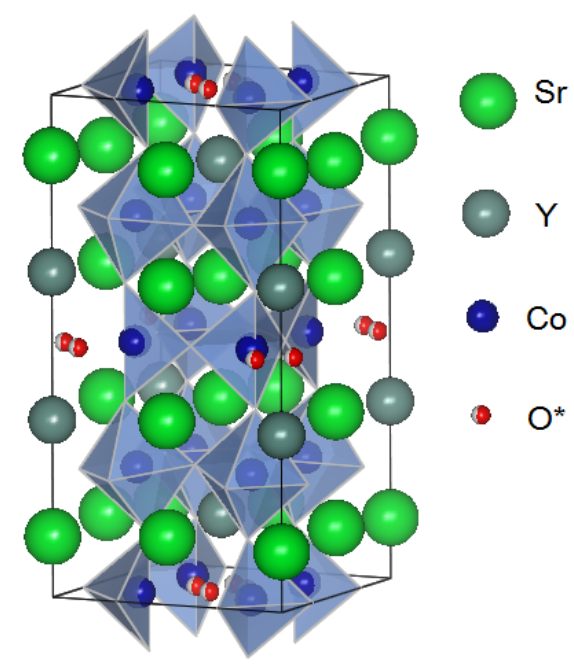

Figure 3 shows the physical properties of the Ca substituted SYCO. In a previous paper [30], we reported the $\mathrm{Ca}$ substitution effects for this compounds below $400 \mathrm{~K}$. Since the $\mathrm{Sr}$ and $\mathrm{Ca}$ ions are divalent, this substitution did not change the oxygen content, but decreased the lattice parameters owing to the smaller ionic radius of $\mathrm{Ca}^{2+}$ ions. As a result, the $\mathrm{Ca}$ substitution acts as chemical pressure, which drives the spin state of $\mathrm{Co}^{3+}$ from the high/intermediate spin state of larger volume to the low spin state of smaller volume, as is similar to the case of $\mathrm{La}_{1-x} R_{x} \mathrm{CoO}_{3}(R=\operatorname{Pr}[35]$ and $\mathrm{Eu}$ [36]). 
Figure 3. The physical properties of the Ca-substituted SYCO. (a) Magnetization $M$ in $0.1 \mathrm{~T}$, (b) resistivity $\rho$ and (c) thermopower $S$.

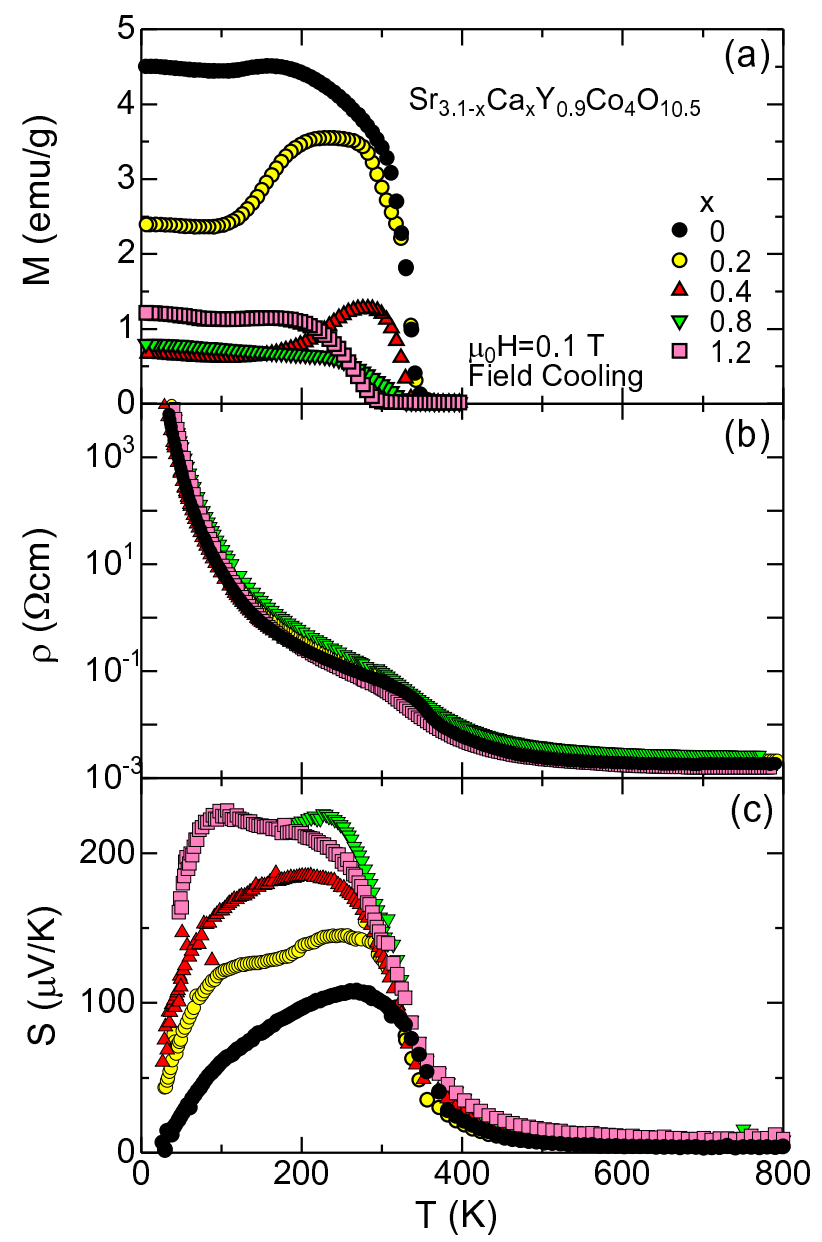

Figure 3(a) shows the the magnetization of $\mathrm{Sr}_{3.1-x} \mathrm{Ca}_{x} \mathrm{Y}_{0.9} \mathrm{Co}_{4} \mathrm{O}_{10.5}$ in $0.1 \mathrm{~T}$. For $x=0$, the magnetization rapidly increases below $340 \mathrm{~K}$, indicating the weak ferromagnetism of this compound. With increasing $\mathrm{Ca}$ content, the magnetization dramatically drops with a decrease in the transition temperature, which is associated with the spin state crossover driven by chemical pressure. It should be noted that the magnetization of $x=0.2$ exhibits complicated behavior. It rises below $340 \mathrm{~K}$, takes a broad maximum around $250 \mathrm{~K}$, and goes down below around $200 \mathrm{~K}$. This indicates the competition between the magnetic order and the spin state crossover. The volume of the $x=0.2$ sample is so critical that the magnetic order becomes unstable below the transition temperature, and some fractions of the $\mathrm{Co}^{3+}$ ions go to the low spin state. For higher substitution, the majority of the $\mathrm{Co}^{3+}$ ions is already in the low spin state at the transition temperature. Owing to unavoidable inhomogeneity due to the solid solution between $\mathrm{Sr}$ and $\mathrm{Ca}$, the chemical pressure is somehow inhomogeneous, and a small amount of magnetization survives up to $x=1.2$.

Figure 3(b) shows the resistivity of $\mathrm{Sr}_{3.1-x} \mathrm{Ca}_{x} \mathrm{Y}_{0.9} \mathrm{Co}_{4} \mathrm{O}_{10.5}$. As is clearly seen, the resistivity is almost independent of the Ca substitution. At $800 \mathrm{~K}$, the resistivity is as low as 2-3 $\mathrm{m} \Omega \mathrm{cm}$, where the $d$ electrons on the $\mathrm{Co}^{3+}$ ion become itinerant. Kobayashi et al. [29] measured the Hall coefficient of $x=0$ in this temperature range and found that the magnitude is of the order of $10^{-4} \mathrm{~cm}^{3} / \mathrm{C}$, which corresponds 
to a carrier density of conventional metals. In this temperature range, all the $\mathrm{Co}^{3+}$ ions are magnetic and metallic, and the decrease of the unit cell volume by the Ca substitution negligibly affects the magnitude of the resistivity. This situation is similar to the high temperature transport in $\mathrm{LaCoO}_{3}$ [37], although the microscopic mechanism is still controversial at present.

The resistivity increases with decreasing temperature, and takes a cusp at the magnetic transition temperature. Since the magnetic $\mathrm{Co}^{3+}$ ions undergo a long range order, they cease to be itinerant, which is detected by an increase of the magnitude of the Hall coefficient [29]. Instead, a small amount of $\mathrm{Co}^{4+}$ ions due to oxygen nonstoichiometry are responsible for electrical conduction. Again, the resistivity is expected to be independent of the Ca content, because the oxygen nonstoichiometry and the content of the $\mathrm{Co}^{4+}$ ions are independent of the Ca content.

In contrast to the resistivity, the thermopower dramatically changes with the Ca content. Figure 3(c) shows the thermopower of $\mathrm{Sr}_{3.1-x} \mathrm{Ca}_{x} \mathrm{Y}_{0.9} \mathrm{Co}_{4} \mathrm{O}_{10.5}$. At high temperature around $800 \mathrm{~K}$, the thermopower is of the order of $1 \mu \mathrm{V} / \mathrm{K}$, which is a typical magnitude for the thermopower of conventional metals. This is consistent with the fact that all the $\mathrm{Co}^{3+}$ ions become itinerant at such temperatures. Toward the transition temperature, the thermopower rapidly increases, suggesting the reduction of the carrier concentration. Below about $300 \mathrm{~K}$, the thermopower exhibits strong Ca dependence. With increasing Ca content, the thermopower largely increases. At $100 \mathrm{~K}$, the thermopower for $x=0$ is $60 \mu \mathrm{V} / \mathrm{K}$, whereas that for $x=1.2$ is $220 \mu \mathrm{V} / \mathrm{K}$. Since the resistivity is essentially the same value between $x=0$ and $x=1.2$, the thermoelectric power factor $S^{2} / \rho$ and perhaps the thermoelectric figure of merit $Z=S^{2} / \rho \kappa$ are enhanced by a factor of $(220 / 60)^{2} \sim 13$. We notice that the resistivity is too high for practical applications, but nevertheless this is a good example that the thermopower can be enhanced with remaining the resistivity unchanged.

This thermopower enhancement is understandable in terms of the spin-state crossover driven by the chemical pressure. In Equation (4), let the $\mathrm{A}$ and $\mathrm{B}$ ions be $\mathrm{Co}^{4+}$ and $\mathrm{Co}^{3+}$, respectively. Then the degeneracy $g_{B}$ is 1 and 15 , respectively, for the low and high spin states of $\mathrm{Co}^{3+}$. Here we used the spin number of $S=2$ and the orbital number of $L=1$ for the high spin state of $\mathrm{Co}^{3+}\left[\left(e_{g}\right)^{2}\left(t_{2 g}\right)^{4}\right]$. We can always assume that $\mathrm{Co}^{4+}$ is in the low spin state $\left(g_{A}=6\right)$. Given a constant $x$, we thus expect that the thermopower should change by $\left(k_{B} \ln 15\right) / e=230 \mu \mathrm{V} / \mathrm{K}$ when the $\mathrm{Co}^{3+}$ ions experience the crossover from the high to low spin state. This value is consistent with the observed value of $220-60=160 \mu \mathrm{V} / \mathrm{K}$, assuming that about $70 \%$ of the $\mathrm{Co}^{3+}$ ions go to the low spin state. It should be emphasized that the resistivity is not affected by the spin state crossover of $\mathrm{Co}^{3+}$. The electric charge is carried with the $\mathrm{Co}^{4+}$ ions, where the $\mathrm{Co}^{3+}$ ions work only as the background. On the other hand, when the background has a finite entropy, the back flow of the background entropy influences the thermopower $[24,38]$.

\subsection{Perovskite rhodium oxide}

The perovskite cobalt oxide $\mathrm{LaCoO}_{3}$ has been extensively studied as a possible thermoelectric material [39-44]. Androulakis et al. [40] reported that slightly doped $\mathrm{LaCoO}_{3}$ is as good as a polycrystalline sample of $\mathrm{Na}_{x} \mathrm{CoO}_{2}$ at room temperature. Robert et al. [41] extensively investigated the thermoelectric properties of doped $\mathrm{RCoO}_{3}$. They found that the $Z T$ values can be improved by properly choosing the rare earth element $R$. Iwasaki et al. [42] comprehensively studied the thermoelectric 
properties of Sr-substituted $\mathrm{LaCoO}_{3}$ from 4 to $1100 \mathrm{~K}$, and found that the thermopower rapidly decreases above $500 \mathrm{~K}$ for all samples. One serious drawback of this class of materials is that the $\mathrm{Co}^{3+}$ ions change their spin state from the low spin to the intermediate/high spin state at high temperatures, and become itinerant like conventional metals [3,37]. Owing to this, the thermopower goes down to a small value of the order of $1 \mu \mathrm{V} / \mathrm{K}$, and decreases $Z T$ at high temperatures. Similar behavior is already seen in SYCO in Figure 3, where the thermopower drops rapidly above around $350 \mathrm{~K}$. Note that the drop occurs at lower temperature than in the case of $\mathrm{LaCoO}_{3}$, because SYCO has a larger unit cell to accept intermediate/high spin state from lower temperatures.

To overcome this drawback, we focus on rhodium oxides. Rhodium is located below cobalt in the periodic table, and thus is expected to have similar chemical properties. In fact, many cobalt oxides have their isomorphic rhodium oxides, and similar transport properties are reported [45-50]. An important difference from the $\mathrm{Co}^{3+}$ ions is that the $\mathrm{Rh}^{3+}$ ions are stable in the low spin state at all the temperatures of interest.

Shibasaki et al. [51] experimentally showed that Ni-substituted $\mathrm{LaRhO}_{3}$ exhibits large thermopower at high temperatures, and has better thermoelectric performance at $800 \mathrm{~K}$. Figure 4 shows the resistivity and thermopower of polycrystalline samples of $\mathrm{LaRh}_{1-x} \mathrm{Ni}_{x} \mathrm{O}_{3}$. Both quantities systematically change with increasing Ni content, showing that the substituted Ni ion acts as an acceptor. They think that the $\mathrm{Ni}$ ions are doped as divalent in the lightly doped region, and induces $\mathrm{Rh}^{4+}$ per $\mathrm{Ni}^{2+}$ to keep the formal valence of the B-site ion to be trivalent (i.e., $2 \mathrm{Rh}^{3+} \rightarrow \mathrm{Rh}^{4+}+\mathrm{Ni}^{2+}$ ), They verified this idea by measuring the susceptibility.

Figure 4. The transport properties of the $\mathrm{LaRh}_{1-x} \mathrm{Ni}_{x} \mathrm{O}_{3}$ [51]. (a) Resistivity and (b) thermopower.

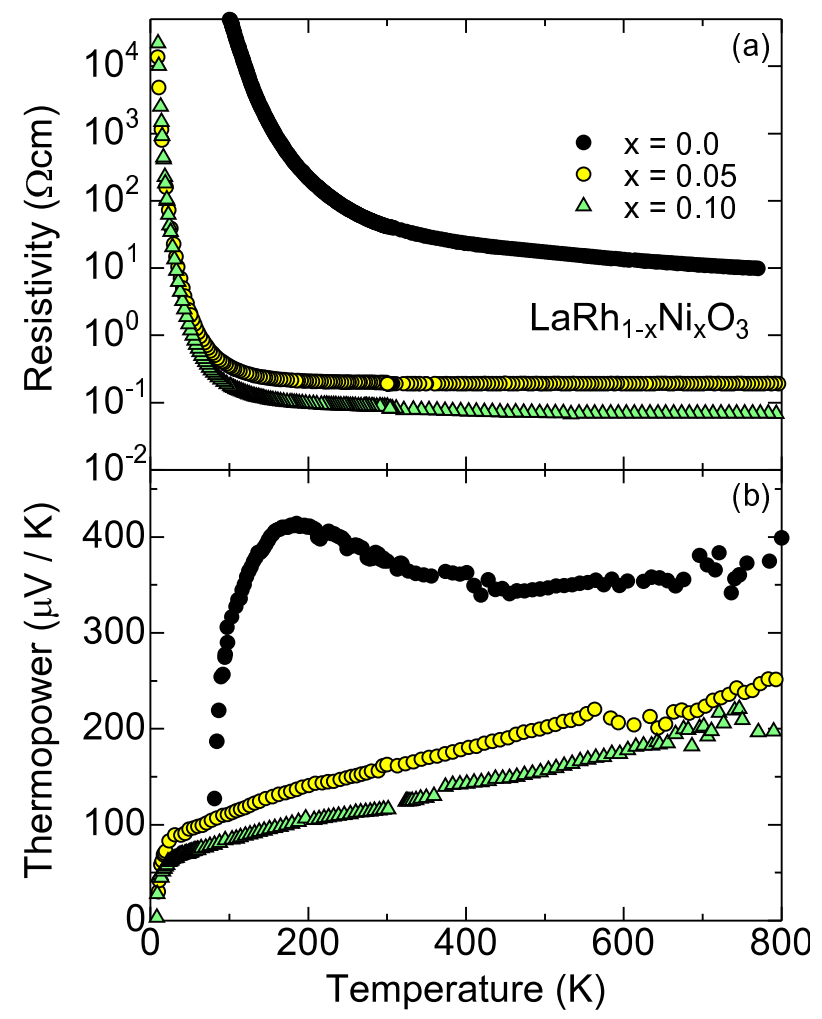


One can see that the thermopower remains large values up to $800 \mathrm{~K}$. This makes a remarkable contrast to the thermopower of $R \mathrm{Co}_{1-x} \mathrm{Ni}_{x} \mathrm{O}_{3}$, where the thermopower rapidly decreases at high temperatures owing to the spin state crossover [41]. This large thermopower is consistent with the fact that $\mathrm{Rh}^{3+}$ is stable in the low spin state. In compensation for the large thermopower, the resistivity is higher than that of $R \mathrm{Co}_{1-x} \mathrm{Ni}_{x} \mathrm{O}_{3}$ [41,44]. $d$ electrons on the $\mathrm{Co}^{3+}$ sites become itinerant at high temperature, and probably conduct in the wide $e_{g}$ bands [37], whereas the conduction occurs always in the narrow $t_{2 g}$ bands in doped $\mathrm{LaRhO}_{3}$. The overlap between the neighboring $t_{2 g}$ bands is smaller in the perovskite structure than in the $\mathrm{CdI}_{2}$-type $\mathrm{CoO}_{2}$ block in $\mathrm{Na}_{x} \mathrm{CoO}_{2}$.

Compared with the B site substitution, the A site substitution is easy to control the formal valence of $\mathrm{Rh}$. Figure 5 shows the resistivity and thermopower of polycrystalline samples of $\mathrm{La}_{1-x} \mathrm{Sr}_{x} \mathrm{RhO}_{3}$. As is similar to Figure 4, both quantities systematically decrease with increasing $\mathrm{Sr}$ content, showing that the doped Sr ion acts as an acceptor. The resistivity shows a metal-insulator transition around $x=0.15$. The critical concentration of $x=0.15$ is significantly smaller than that of $\mathrm{La}_{1-x} \mathrm{Sr}_{x} \mathrm{CoO}_{3}(\sim 0.3)$ [52]. In $\mathrm{La}_{1-x} \mathrm{Sr}_{x} \mathrm{RhO}_{3}$, the electrical conduction occurs only in the $t_{2 g}$ bands, and both of $\mathrm{Rh}^{3+}$ and $\mathrm{Rh}^{4+}$ are in the low spin states. In $\mathrm{La}_{1-x} \mathrm{Sr}_{x} \mathrm{CoO}_{3}$, on the other hand, the doped $\mathrm{Co}^{4+}$ induces the spin state crossover to the neighboring $\mathrm{Co}^{3+}$ to make a spin polaron [53]. The spin polaron can be itinerant at room temperature, where most of the $\mathrm{Co}^{3+}$ ions are magnetic. With decreasing temperature, the low spin state becomes stable for the $\mathrm{Co}^{3+}$ ions away from the $\mathrm{Co}^{4+}$ ions, and as a result, electronic phase separation takes place to localize the spin polaron [54]. As such, the resistivity goes nonmetallic below $x=0.3$ in $\mathrm{La}_{1-x} \mathrm{Sr}_{x} \mathrm{CoO}_{3}$ at low temperatures.

Figure 5. The transport properties of the $\mathrm{La}_{1-x} \mathrm{Sr}_{x} \mathrm{RhO}_{3}$. (a)Resistivity and (b)thermopower.

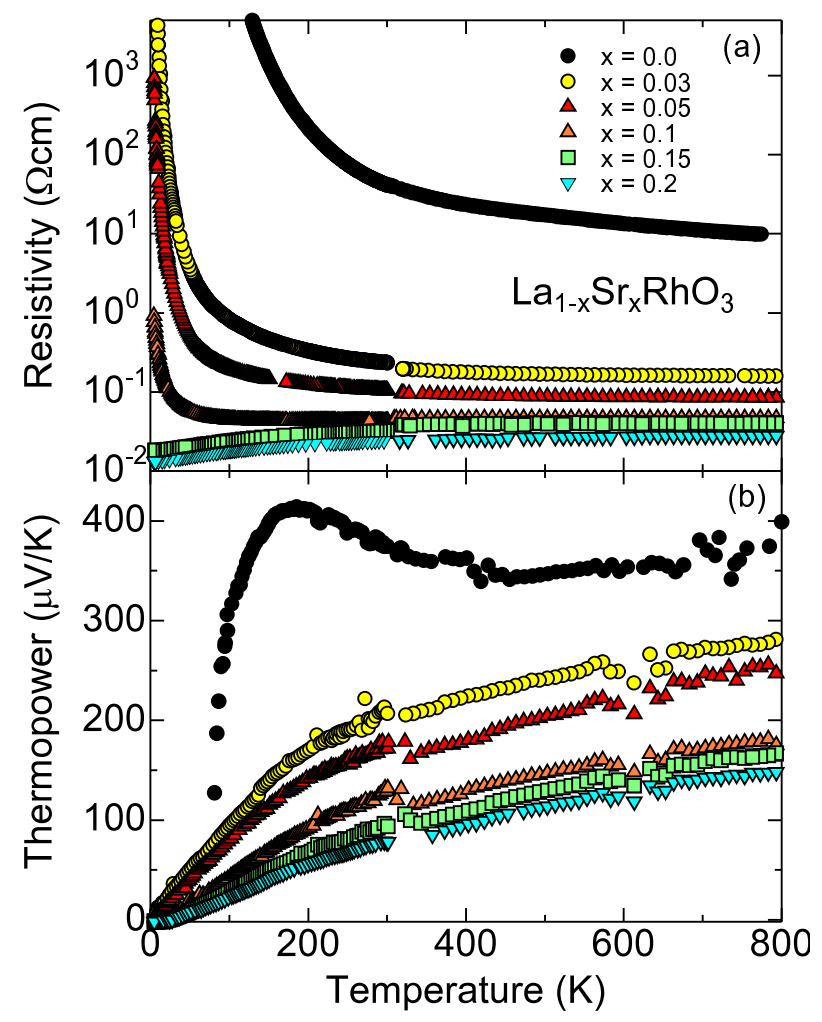


The thermopower also make a remarkable contrast to those of $\mathrm{La}_{1-x} \mathrm{Sr}_{x} \mathrm{CoO}_{3}$ [42]. The thermopower continues to increase up to $800 \mathrm{~K}$ for all $x$, and remains larger than $150 \mu \mathrm{V} / \mathrm{K}$ at $800 \mathrm{~K}$, as is already discussed with Equation (4). Although Equation (4) is based on a picture of the localized electrons (strong correlation picture), the temperature dependence seen in Figure 5 is like that of degenerate semiconductors. This suggests that the electronic state is basically understood by a band picture (moderate correlation picture). Actually, when $\mathrm{Rh}^{3+}$ and $\mathrm{Rh}^{4+}\left(\mathrm{Co}^{3+}\right.$ and $\left.\mathrm{Co}^{4+}\right)$ are both in the low spin states, the electrical conduction may be explained from the band theory because of the absence of local moments. At an early stage of the thermoelectric study in $\mathrm{Na}_{x} \mathrm{CoO}_{2}$, Singh [55] already pointed out that the thermopower and specific heat of $\mathrm{Na}_{x} \mathrm{CoO}_{2}$ can be quantitatively understood from an LDA calculation. Recently Usui et al. [56] have calculated the thermopower of doped $\mathrm{LaRhO}_{3}$ using an ab-initio calculation, which quantitatively agrees with the room-temperature thermopower in Figure 4.

It has been a long-standing problem in conducting transition metal oxides which picture (localized or itinerant electron picture) describes better (e.g., see [57]). Thus it may suffice to say that there exist some anomalous features beyond simple band pictures. One feature is that the thermopower of the Ni-substituted $\mathrm{LaRhO}_{3}$ shown in Figure 4 has a peculiar cusp around $20 \mathrm{~K}$. This is different from the thermopower of conventional metals and semiconductors, and seems difficult to be calculated. Empirically, such temperature dependence is seen in disordered $\mathrm{Co} / \mathrm{Rh}$ oxides such as $\mathrm{B}$-site substituted $\mathrm{LaCoO}_{3}$ [43], $\mathrm{Ca}_{3} \mathrm{Co}_{4} \mathrm{O}_{9}$ [20,58], Bi-Sr-Co-O [21,59], and Bi-Sr-Rh-O [45,47]. A second feature is the nontrivial magnetism of the perovskite rhodium oxides; $\mathrm{SrRhO}_{3}$ is an antiferromagnetic metal [60] and $\mathrm{La}_{1-x} \mathrm{Sr}_{x} \mathrm{RhO}_{3}$ is a Curie-Weiss metal [61]. This indicates that $\mathrm{Rh}^{4+}$ (and possibly a mixture of $\mathrm{Rh}^{3+}$ and $\mathrm{Rh}^{4+}$ as well) is magnetic, which is difficult to predict from the band calculation.

\section{Experimental Section}

Polycrystalline samples were prepared by a solid-state reaction method. $\mathrm{For}_{\mathrm{Sr}_{3}} \mathrm{YCo}_{4} \mathrm{O}_{10.5}, \mathrm{SrCO}_{3}$, $\mathrm{CaCO}_{3}, \mathrm{Y}_{2} \mathrm{O}_{3}$ and $\mathrm{Co}_{3} \mathrm{O}_{4}$ were mixed and calcined at $1,100^{\circ} \mathrm{C}$ for $12 \mathrm{~h}$ in air. In order to compensate the evaporation of Co during sintering, we deliberately added an excess 5-mol\% Co as starting composition, i.e., the nominal composition was set to be $\mathrm{Sr}_{3.12-x} \mathrm{Ca}_{x} \mathrm{Y}_{0.88} \mathrm{Co}_{4.2} \mathrm{O}_{y}$. For further details, see the reference [30]. The calcined product was ground, pressed into a pellet, and sintered at $1,100{ }^{\circ} \mathrm{C}$ for $48 \mathrm{~h}$ in air. For $\mathrm{LaRh}_{1-x} \mathrm{Ni}_{x} \mathrm{O}_{3}$ and $\mathrm{La}_{1-x} \mathrm{Sr}_{x} \mathrm{RhO}_{3}$, stoichiometric amounts of $\mathrm{La}_{2} \mathrm{O}_{3}, \mathrm{SrCO}_{3}, \mathrm{Rh}_{2} \mathrm{O}_{3}$ and $\mathrm{NiO}$ were mixed, and calcined at $1,000{ }^{\circ} \mathrm{C}$ for $24 \mathrm{~h}$ in air. The calcined products were thoroughly ground, pelletized and sintered at $1,100-1,200{ }^{\circ} \mathrm{C}$ for $48 \mathrm{~h}$ in air.

The prepared ceramic samples were characterized by an X-ray diffractometer with a $\theta-2 \theta$ scan mode, and were verified to be in single phase with no detectable impurities. The magnetization-temperature curves were measured using a commercial superconducting quantum interference device magnetometer (Quantum Design MPMS) in a field cooling process of $0.1 \mathrm{~T}$. The resistivity was measured using a four-probe method and the thermopower was measured with a steady-state method with a typical temperature gradient of $1 \mathrm{~K} / \mathrm{cm}$. The resistivity and thermopower were measured in a liquid He cryostat below room temperature, and were measured in vacuum on a sapphire substrate painted with $\mathrm{RuO}_{2}$ paste used as a resistive heater above room temperature. 


\section{Summary}

In this article, we have shown the thermoelectric properties of the two perovskite-related oxides, and discuss the relationship to the spin states. In the A-site ordered cobalt oxide $\mathrm{Sr}_{3} \mathrm{YCo}_{4} \mathrm{O}_{10.5}$, partial substitution of $\mathrm{Ca}$ for $\mathrm{Sr}$ acting as chemical pressure enhances the low-temperature thermopower without increasing resistivity appreciably. This is understood in terms of the spin state crossover driven by the chemical pressure. When the background $\mathrm{Co}^{3+}$ ions go to the low spin state, the entropy flow by the carrier on the $\mathrm{Co}^{4+}$ ion changes, and concomitantly the thermopower changes. In the perovskite rhodium oxide $\mathrm{La}_{1-x} \mathrm{Sr}_{x} \mathrm{RhO}_{3}$, the thermopower remains large up to high temperatures ( $>150 \mu \mathrm{V} / \mathrm{K}$ at $800 \mathrm{~K}$ ), which makes a remarkable contrast to $\mathrm{La}_{1-x} \mathrm{Sr}_{x} \mathrm{CoO}_{3}$. This is associated with the stability of the low spin state of $\mathrm{Rh}^{3+}$ ions. Through the two examples, we suggest that the spin state control is a unique and effective tool for oxide thermoelectrics.

\section{Acknowledgements}

We would like to S. Ishiwata, M. Karppinen, H. Yamauchi, S. Kimura, M. Hagiwara, H. Nakao, Y. Murakami for collaboration, and A. Weidenkaff, A. Maignan, S. Hébert, K. Kuroki, D. J. Singh for fruitful discussion. This work is partially supported by MEXT, Japan (Nos. 16076213 and 21340106).

\section{References}

1. Sugano, S.; Tanabe, Y.; Kamimura, H. Multiplets of Transition-Metal Ions in Crystals; Academic Press: New York, NY, USA, 1970.

2. Gütlich, P.; Garcia, Y.; Goodwin, H.A. Spin crossover phenomena in Fe(II) complexes. Chem. Soc. Rev. 2000, 29, 419-427.

3. Raccah, P.M.; Goodenough, J.B. First-order localized-electron collective-electron transition in $\mathrm{LaCoO}_{3}$. Phys. Rev. 1967, 155, 932-943.

4. Asai, K.; Yoneda, A.; Yokokura, O.; Tranquada, J.M.; Shirane, G.; Kohn, K. Two spin-state transitions in $\mathrm{LaCoO}_{3}$. J. Phys. Soc. Jpn. 1998, 67, 290-296.

5. Vogt, T.; Hriljac, J.A.; Hyatt, N.C.; Woodward, P. Pressure-induced intermediate-to-low spin state transition in $\mathrm{LaCoO}_{3}$. Phys. Rev. B 2003, 67, 140401.

6. Korotin, M.A.; Ezhov, S.Y.; Solovyev, I.V.; Anisimov, V.I.; Khomskii, D.I.; Sawatzky, G.A. Intermediate-spin state and properties of $\mathrm{LaCoO}_{3}$. Phys. Rev. B 1996, 54, 5309-5316.

7. Noguchi, S.; Kawamata, S.; Okuda, K.; Nojiri, H.; Motokawa, M. Evidence for the excited triplet of $\mathrm{Co}^{3+}$ in $\mathrm{LaCoO}_{3}$. Phys. Rev. B 2002, 66, 094404.

8. Maris, G.; Ren, Y.; Volotchaev, V.; Zobel, C.; Lorenz, T.; Palstra, T.T.M. Evidence for orbital ordering in $\mathrm{LaCoO}_{3}$. Phys. Rev. B 2003, 67, 224423.

9. Haverkort, M.W.; Hu, Z.; Cezar, J.C.; Burnus, T.; Hartmann, H.; Reuther, M.; Zobel, C.; Lorenz, T.; Tanaka, A.; Brookes, N.B.; Hsieh, H.H.; Lin, H.J.; Chen, C.T.; Tjeng, L.H. Spin state transition in $\mathrm{LaCoO}_{3}$ studied using soft X-ray absorption spectroscopy and magnetic circular dichroism. Phys. Rev. Lett. 2006, 97, 176405. 
10. Klie, R.F.; Zheng, J.C.; Zhu, Y.; Varela, M.; Wu, J.; Leighton, C. Direct measurement of the low-temperature spin-state transition in $\mathrm{LaCoO}_{3}$. Phys. Rev. Lett. 2007, 99, 047203.

11. Terasaki, I.; Sasago, Y.; Uchinokura, K. Large thermoelectric power in $\mathrm{NaCo}_{2} \mathrm{O}_{4}$ single crystals. Phys. Rev. B 1997, 56, R12685-R12687.

12. Koumoto, K.; Terasaki, I.; Funahashi, R. Complex oxide materials for potential thermoelectric applications. MRS Bull. 2006, 31, 206-210.

13. Terasaki, I. Transport properties and electronic states of the thermoelectric oxide $\mathrm{NaCo}_{2} \mathrm{O}_{4}$. Physica B 2003, 328, 63-67.

14. Koshibae, W.; Tsutsui, K.; Maekawa, S. Thermopower in cobalt oxides. Phys. Rev. B 2000, 62, 6869-6872.

15. Callen, H.B. Thermodynamics and an Introduction to Thermostatistics, 2nd ed.; John Wiley \& Sons, Inc.: Hoboken, NJ, USA, 1985.

16. Chaikin, P.M.; Beni, G. Thermopower in the correlated hopping regime. Phys. Rev. B 1976, 13, 647-651.

17. Ray, R.; Ghoshray, A.; Ghoshray, K.; Nakamura, S. ${ }^{59}$ Co NMR studies of metallic $\mathrm{NaCo}_{2} \mathrm{O}_{4}$. Phys. Rev. B 1999, 59, 9454-9461.

18. Fujita, K.; Mochida, T.; Nakamura, K. High-temperature thermoelectric properties of $\mathrm{Na}_{x} \mathrm{CoO}_{2-\delta}$ Single Crystals. Jpn. J. Appl. Phys. 1 2001, 40, 4644-4647.

19. Ando, Y.; Miyamoto, N.; Segawa, K.; Kawata, T.; Terasaki, I. Specific-heat evidence for strong electron correlations in the thermoelectric material $(\mathrm{Na}, \mathrm{Ca}) \mathrm{Co}_{2} \mathrm{O}_{4}$. Phys. Rev. B 1999, 60, 10580-10583.

20. Masset, A.C.; Michel, C.; Maignan, A.; Hervieu, M.; Toulemonde, O.; Studer, F.; Raveau, B.; Hejtmanek, J. Misfit-layered cobaltite with an anisotropic giant magnetoresistance: $\mathrm{Ca}_{3} \mathrm{Co}_{4} \mathrm{O}_{9}$. Phys. Rev. B 2000, 62, 166-175.

21. Maignan, A.; Wang, L.B.; Hébert, S.; Pelloquin, D.; Raveau, B. Large thermopower in metallic misfit cobaltites. Chem. Mater. 2002, 14, 1231-1235.

22. Funahashi, R.; Matsubara, I.; Ikuta, H.; Takeuchi, T.; Mizutani, U.; Sodeoka, S. An oxide single crystal with high thermoelectric performance in air. Jpn. J. Appl. Phys. 2000, 39, L1127-L1129.

23. Funahashi, R.; Shikano, M. $\mathrm{Bi}_{2} \mathrm{Sr}_{2} \mathrm{Co}_{2} \mathrm{O}_{y}$ whiskers with high thermoelectric figure of merit. Appl. Phys. Lett. 2002, 81, 1459-1461.

24. Kobayashi, W.; Terasaki, I.; Mikami, M.; Funahashi, R. Negative thermoelectric power induced by positive carriers in $\mathrm{CaMn}_{3-x} \mathrm{Cu}_{x} \mathrm{Mn}_{4} \mathrm{O}_{12}$. J. Phys. Soc. Jpn. 2004, 73, 523-525.

25. Kobayashi, W.; Ishiwata, S.; Terasaki, I.; Takano, M.; Grigoraviciute, I.; Yamauchi, H.; Karppinen, M. Room-temperature ferromagnetism in $\mathrm{Sr}_{1-x} \mathrm{Y}_{x} \mathrm{CoO}_{3-\delta}(0.2 \leq x \leq 0.25)$. Phys. Rev. B 2005, $72,104408$.

26. Istomin, S.Y.; Grins, J.; Svensson, G.; Drozhzhin, O.A.; Kozhevnikov, V.L.; Antipov, E.V.; Attfield, J.P. Crystal structure of the novel complex cobalt oxide $\mathrm{Sr}_{0.7} \mathrm{Y}_{0.3} \mathrm{CoO}_{2.62}$. Chem. Mater. 2003, 15, 4012-4020.

27. James, M.; Cassidy, D.; Goossens, D.; Withers, R. The phase diagram and tetragonal superstructures of the rare earth cobaltate phases $\mathrm{Ln}_{1-x} \mathrm{Sr}_{x} \mathrm{CoO}_{3-\delta}\left(\mathrm{Ln}=\mathrm{La}^{3+}, \mathrm{Pr}^{3+}, \mathrm{Nd}^{3+}, \mathrm{Sm}^{3+}\right.$, $\mathrm{Gd}^{3+}, \mathrm{Y}^{3+}, \mathrm{Ho}^{3+}, \mathrm{Dy}^{3+}, \mathrm{Er}^{3+}, \mathrm{Tm}^{3+}$ and $\left.\mathrm{Yb}^{3+}\right)$. J. Solid State Chem. 2004, 177, 1886-1895. 
28. Withers, R.L.; James, M.; Goossens, D.J. Atomic ordering in the doped rare earth cobaltates $\mathrm{Ln}_{0.33} \mathrm{Sr}_{0.67} \mathrm{CoO}_{3-\delta}\left(\mathrm{Ln}=\mathrm{Y}^{3+}, \mathrm{Ho}^{3+}\right.$ and $\left.\mathrm{Dy}^{3+}\right)$. J. Solid State Chem. 2003, 174, 198-208.

29. Kobayashi, W.; Yoshida, S.; Terasaki, I. High-temperature metallic state of room-temperature ferromagnet $\mathrm{Sr}_{1-x} \mathrm{Y}_{x} \mathrm{CoO}_{3-\delta}$. J. Phys. Soc. Jpn. 2006, 75, 103702.

30. Yoshida, S.; Kobayashi, W.; Nakano, T.; Terasaki, I.; Matsubayashi, K.; Uwatoko, Y.; Grigoraviciute, I.; Karppinen, M.; Yamauchi, H. Chemical and physical pressure effects on the magnetic and transport properties of the A-site ordered perovskite $\mathrm{Sr}_{3} \mathrm{YCo}_{4} \mathrm{O}_{10.5}$. J. Phys. Soc. Jpn. 2009, 78, 094711.

31. Kimura, S.; Maeda, Y.; Kashiwagi, T.; Yamaguchi, H.; Hagiwara, M.; Yoshida, S.; Terasaki, I.; Kindo, K. Field-induced spin-state transition in the perovskite cobalt oxide $\mathrm{Sr}_{1-x} \mathrm{Y}_{x} \mathrm{CoO}_{3-\delta}$. Phys. Rev. B 2008, 78, 180403.

32. Sheptyakov, D.V.; Pomjakushin, V.Y.; Drozhzhin, O.A.; Istomin, S.Y.; Antipov, E.V.; Bobrikov, I.A.; Balagurov, A.M. Correlation of chemical coordination and magnetic ordering in $\mathrm{Sr}_{3} \mathrm{YCo}_{4} \mathrm{O}_{10.5+\delta}(\delta=0.02$ and 0.26). Phys. Rev. B 2009, 80, 024409.

33. Ishiwata, S.; Kobayashi, W.; Terasaki, I.; Kato, K.; Takata, M. Structure-property relationship in the ordered-perovskite-related oxide $\mathrm{Sr}_{3.12} \mathrm{Er}_{0.88} \mathrm{Co}_{4} \mathrm{O}_{10.5}$. Phys. Rev. B 2007, 75, 220406.

34. James, M.; Avdeev, M.; Barnes, P.; Morales, L.; Wallwork, K.; Withers, R. Orthorhombic superstructures within the rare earth strontium-doped cobaltate perovskites: $L n_{1-x} \mathrm{Sr}_{x} \mathrm{CoO}_{3-d}$ $\left(\right.$ Ln $\left.=\mathrm{Y}^{3+}, \mathrm{Dy}^{3+}-\mathrm{Yb}^{3+} ; 0.750 \leq x \leq 0.875\right)$. J. Solid State Chem. 2007, 180, 2233-2247.

35. Kobayashi, Y.; Mogi, T.; Asai, K. Spin-state transition in $\mathrm{La}_{1-x} \operatorname{Pr}_{x} \mathrm{CoO}_{3}$. J. Phys. Soc. Jpn. 2006, $75,104703$.

36. Baier, J.; Jodlauk, S.; Kriener, M.; Reichl, A.; Zobel, C.; Kierspel, H.; Freimuth, A.; Lorenz, T. Spin-state transition and metal-insulator transition in $\mathrm{La}_{1-x} \mathrm{Eu}_{x} \mathrm{CoO}_{3}$. Phys. Rev. B 2005, $71,014443$.

37. Tokura, Y.; Okimoto, Y.; Yamaguchi, S.; Taniguchi, H.; Kimura, T.; Takagi, H. Thermally induced insulator-metal transition in $\mathrm{LaCoO}_{3}$ : A view based on the Mott transition. Phys. Rev. B 1998, 58, R1699-R1702.

38. Palstra, T.T.M.; Ramirez, A.P.; Cheong, S.W.; Zegarski, B.R.; Schiffer, P.; Zaanen, J. Transport mechanisms in doped $\mathrm{LaMnO}_{3}$ : Evidence for polaron formation. Phys. Rev. B 1997, 56, 5104-5107.

39. Hébert, S.; Flahaut, D.; Martin, C.; Lemonnier, S.; Noudem, J.; Goupil, C.; Maignan, A.; Hejtmanek, J. Thermoelectric properties of perovskites: Sign change of the Seebeck coefficient and high temperature properties. Prog. Solid State Chem. 2007, 35, 457-467.

40. Androulakis, J.; Migiakis, P.; Giapintzakis, J. $\mathrm{La}_{0.95} \mathrm{Sr}_{0.05} \mathrm{CoO}_{3}$ : An efficient room-temperature thermoelectric oxide. Appl. Phys. Lett. 2004, 84, 1099-1101.

41. Robert, R.; Aguirre, M.; Hug, P.; Reller, A.; Weidenkaff, A. High-temperature thermoelectric properties of $\mathrm{Ln}(\mathrm{Co}, \mathrm{Ni}) \mathrm{O}_{3}(\mathrm{Ln}=\mathrm{La}, \mathrm{Pr}, \mathrm{Nd}, \mathrm{Sm}, \mathrm{Gd}$ and Dy) compounds. Acta Mater. 2007, $55,4965-4972$.

42. Iwasaki, K.; Ito, T.; Nagasaki, T.; Arita, Y.; Yoshino, M.; Matsui, T. Thermoelectric properties of polycrystalline $\mathrm{La}_{1-x} \mathrm{Sr}_{x} \mathrm{CoO}_{3}$. J. Solid State Chem. 2008, 181, 3145 - 3150. 
43. Jirák, Z.; Hejtmánek, J.; Knívzek, K.; Veverka, M. Electrical resistivity and thermopower measurements of the hole- and electron-doped cobaltites $\mathrm{LnCoO}_{3}$. Phys. Rev. B 2008, 78, 014432.

44. Migiakis, P.; Androulakis, J.; Giapintzakis, J. Thermoelectric properties of $\mathrm{LaNi}_{1-x} \mathrm{Co}_{x} \mathrm{O}_{3}$ solid solution. J. Appl. Phys. 2003, 94, 7616-7620.

45. Klein, Y.; Hébert, S.; Pelloquin, D.; Hardy, V.; Maignan, A. Magnetoresistance and magnetothermopower in the rhodium misfit oxide $\left[\mathrm{Bi}_{1.95} \mathrm{Ba}_{1.95} \mathrm{Rh}_{0.1} \mathrm{O}_{4}\right]\left[\mathrm{RhO}_{2}\right]_{1.8}$. Phys. Rev. B 2006, 73, 165121.

46. Maignan, A.; Eyert, V.; Martin, C.; Kremer, S.; Frésard, R.; Pelloquin, D. Electronic structure and thermoelectric properties of $\mathrm{CuRh}_{1-x} \mathrm{Mg}_{x} \mathrm{O}_{2}$. Phys. Rev. B 2009, 80, 115103.

47. Okada, S.; Terasaki, I. Physical properties of Bi-based rhodium oxides with $\mathrm{RhO}_{2}$ hexagonal layers. Jpn. J. Appl. Phys. 2005, 44, 1834-1837.

48. Okada, S.; Terasaki, I.; Okabe, H.; Matoba, M. Transport properties and electronic states in the layered thermoelectric rhodate $\left(\mathrm{Bi}_{1-x} \mathrm{~Pb}_{x}\right)_{1.8} \mathrm{Ba}_{2} \mathrm{Rh}_{1.9} \mathrm{O}_{y}$. J. Phys. Soc. Jpn. 2005, 74, 1525-1528.

49. Okamoto, Y.; Nohara, M.; Sakai, F.; Takagi, H. Correlated metallic phase in a doped band insulator $\mathrm{Sr}_{1-x} \mathrm{Rh}_{2} \mathrm{O}_{4}$. J. Phys. Soc. Jpn. 2006, 75, 023704.

50. Shibasaki, S.; Kobayashi, W.; Terasaki, I. Transport properties of the delafossite Rh oxide $\mathrm{Cu}_{1-x} \mathrm{Ag}_{x} \mathrm{Rh}_{1-y} \mathrm{Mg}_{y} \mathrm{O}_{2}$ : Effect of $\mathrm{Mg}$ substitution on the resistivity and Hall coefficient. Phys. Rev. B 2006, 74, 235110.

51. Shibasaki, S.; Takahashi, Y.; Terasaki, I. Thermoelectric properties of $\mathrm{LaRh}_{1-x} \mathrm{Ni}_{x} \mathrm{O}_{3}$. J. Phys.: Condens. Matter 2009, 21, 115501.

52. Kriener, M.; Zobel, C.; Reichl, A.; Baier, J.; Cwik, M.; Berggold, K.; Kierspel, H.; Zabara, O.; Freimuth, A.; Lorenz, T. Structure, magnetization, and resistivity of $\mathrm{La}_{1-x} M_{x} \mathrm{CoO}_{3}(M=\mathrm{Ca}, \mathrm{Sr}$, and Ba). Phys. Rev. B 2004, 69, 094417.

53. Podlesnyak, A.; Russina, M.; Furrer, A.; Alfonsov, A.; Vavilova, E.; Kataev, V.; Büchner, B.; Strässle, T.; Pomjakushina, E.; Conder, K.; Khomskii, D.I. Spin-state polarons in lightly-hole-doped $\mathrm{LaCoO}_{3}$. Phys. Rev. Lett. 2008, 101, 247603.

54. Wu, J.; Zheng, H.; Mitchell, J.F.; Leighton, C. Glassy transport phenomena in a phase-separated perovskite cobaltite. Phys. Rev. B 2006, 73, 020404.

55. Singh, D.J. Electronic structure of $\mathrm{NaCo}_{2} \mathrm{O}_{4}$. Phys. Rev. B 2000, 61, 13397-13402.

56. Usui, H.; Arita, R.; Kuroki, K. First-principles study on the origin of large thermopower in hole-doped $\mathrm{LaRhO}_{3}$ and $\mathrm{CuRhO}_{2}$. J. Phys.: Condens. Matter 2009, 21, 064223.

57. Wentzcovitch, R.M.; Schulz, W.W.; Allen, P.B. $\mathrm{VO}_{2}$ : Peierls or Mott-Hubbard? A view from band theory. Phys. Rev. Lett. 1994, 72, 3389-3392.

58. Limelette, P.; Hébert, S.; Hardy, V.; Frésard, R.; Simon, C.; Maignan, A. Scaling behavior in thermoelectric misfit cobalt oxides. Phys. Rev. Lett. 2006, 97, 046601.

59. Itoh, T.; Terasaki, I. Thermoelectric properties of $\mathrm{Bi}_{2.3-x} \mathrm{~Pb}_{x} \mathrm{Sr}_{2.6} \mathrm{Co}_{2} \mathrm{O}_{y}$ single crystals. Jpn. J. Appl. Phys. 1 2000, 39, 6658-6660.

60. Yamaura, K.; Takayama-Muromachi, E. Enhanced paramagnetism of the $4 d$ itinerant electrons in the rhodium oxide perovskite $\mathrm{SrRhO}_{3}$. Phys. Rev. B 2001, 64, 224424. 
61. Nakamura, T.; Shimura, T.; Itoh, M.; Takeda, Y. Magnetic and electric properties of $\mathrm{La}_{1-x} \mathrm{M}_{x} \mathrm{RhO}_{3}$ $(\mathrm{M}=\mathrm{Ca}, \mathrm{Sr}$, and $\mathrm{Ba})$ : Hole doping in $4 d \varepsilon$ orbitals of $\mathrm{Rh}^{3+}$ with low spin configuration. J. Solid State Chem. 1993, 103, 523-527.

(c) 2010 by the authors; licensee Molecular Diversity Preservation International, Basel, Switzerland. This article is an open-access article distributed under the terms and conditions of the Creative Commons Attribution license http://creativecommons.org/licenses/by/3.0/. 